\title{
H-STRUCTURES ON PRODUCTS
}

\author{
ARTHUR H. COPELAND, JR.
}

\begin{abstract}
This note outlines a technique for determining the set of homotopy classes of $H$-structures on a product of $H$-spaces. The technique is then applied to computing the number of $\mathrm{H}$ structures on $S O(4)$.
\end{abstract}

1. Introduction. This note outlines a technique for determining the set of homotopy classes of $H$-structures on a product $X=X_{1} \times X_{2} \times \cdots \times X_{n}$ of $H$-spaces. This technique is then applied to computing the number of $H$-structures on $S O(4)=S O(3) \times S^{3}$.

The technique may be summarized in the following statements.

(1) The set of homotopy classes of $H$-structures on $X$ is in one-to-one correspondence with the homotopy set $[X \wedge X ; X]$.

(2) There are subcomplexes $*=L_{0} \subset L_{1} \subset \cdots \subset L_{2 n}=X \wedge X$ such that the sequences $0 \rightarrow\left[L_{r} / L_{r-1} ; X\right] \rightarrow\left[L_{r} ; X\right] \rightarrow\left[L_{r-1} ; X\right] \rightarrow 0$ are exact for $r=1$, $2, \cdots, 2 n$.

(3) $\left[L_{r} / L_{r-1} ; X\right]$ is the direct product of the loops $\left[X_{i_{1}} \wedge \cdots \wedge X_{i_{p}} \wedge\right.$ $\left.X_{j_{1}} \wedge \cdots \wedge X_{j_{q}} ; X\right]$ with $p, q \geqq 1$ and $p+q=r$.

(4) For any pointed space $L,[L ; X]$ is the direct product of the loops $\left[L ; X_{k}\right]$ for $k=1,2, \cdots, n$.

Statement (4) is completely routine and (1) is essentially Theorem 5.5A of [1]. Statements (2) and (3) are established in $\$ 2$. Throughout this note all spaces should be assumed to be $\mathrm{CW}$-complexes of finite type.

As a corollary to the statements (1)-(4) above, one sees that the number $\#[X \wedge X ; X]$ of products on $X$ is the product of the numbers

$$
\#\left[X_{i_{1}} \wedge \cdots \wedge X_{i_{p}} \wedge X_{j_{1}} \wedge \cdots \wedge X_{j_{q}} ; X_{k}\right] .
$$

2. Main results. We assume that each of the spaces $X_{k}(k=1,2, \cdots, n)$ is a path-connected, pointed CW-complex of finite type and has at least one $H$-structure. As usual, * will be used ambiguously to denote basepoints. Let $X=X_{1} \times \cdots \times X_{n}$ and let $\phi: X \times X \rightarrow X \wedge X$ be the usual identification map. Suppose $p, q$ are integers with $1 \leqq p, q \leqq n$ and that $\alpha=$ $\left(i_{1}, \cdots, i_{p}, j_{1}, \cdots, j_{q}\right)$ is a $(p+q)$-tuple of integers with $1 \leqq i_{1}<i_{2}<\cdots<i_{p} \leqq n$

Received by the editors March 2, 1971.

AMS 1970 subject classifications. Primary 55D45.

Key words and phrases. $H$-spaces, $H$-products, products of $H$-spaces, retractile subcomplexes, cofibration sequence.

(c) American Mathematical Society 1972 
and $1 \leqq j_{1}<j_{2}<\cdots<j_{q} \leqq n$. Such an $\alpha$ will be called admissible. Define $\# \alpha=p+q$ and let $K_{\alpha} \subset X \wedge X$ be the set of points $\phi\left(x_{1}, \cdots, x_{n}, x_{1}^{\prime}, \cdots, x_{n}^{\prime}\right)$ with $x_{i}=x_{j}=*$ except when $i=i_{1}, \cdots, i_{p}$ or $j=j_{1}, \cdots, j_{q}$. Let $L_{r}=$ $\bigcup\left\{K_{\alpha} \mid \# \alpha=r\right\}$ for $r=2,3, \cdots, 2 n$ and let $L_{1}=*=\phi(X \vee X)$. Note that $L_{1} \subset L_{2} \subset \cdots \subset L_{2 n}=X \wedge X$. Let $M_{\alpha}=X_{i_{1}} \wedge \cdots \wedge X_{i_{p}} \wedge X_{j_{1}} \wedge \cdots \wedge X_{j_{q}}$.

2.1 LEMMA (JAMES). If $Y_{1}, \cdots, Y_{m}$ are subcomplexes of $Y$ and if $r_{i}: Y \rightarrow Y_{i}$ are retractions with $r_{i}\left(Y_{j}\right) \subset Y_{j}$ for all $i$ and $j$, then $Y_{1} \cup \cdots \cup Y_{m}$ is retractile in $Y$ [3, Lemma 3.1].

2.2 LEMMA. If $r \leqq s$, then $L_{r}$ is retractile in $L_{s}$.

Proof. In view of [8, Proposition (3.7)] it suffices to show each $L_{r}$ is retractile in $X \wedge X$. But the projections

$$
X \times X \rightarrow X_{i_{1}} \times \cdots \times X_{i_{p}} \times X_{j_{1}} \times \cdots \times X_{j_{q}}
$$

induce retractions $X \wedge X \rightarrow K_{\alpha}$ satisfying the hypothesis of James' Lemma.

2.3 THEOREM. The sequences

$$
0 \rightarrow\left[L_{r} / L_{r-1} ; X\right] \rightarrow\left[L_{r} ; X\right] \rightarrow\left[L_{r-1} ; X\right] \rightarrow 0
$$

are exact for $r=2,3, \cdots, 2 n$.

This is an immediate consequence of O'Neill's work [8, Theorems (3.3) and (3.4)]. Note that if $X$ is the space of loops on an $H$-space, then a result of Eckmann and Hilton [2, Theorem 2.2] implies that these sequences split.

2.4 THEOREM. $\left[L_{r} / L_{r-1} ; X\right]$ is the direct product of the loops $\left[M_{\alpha} ; X\right]$ with $\# \alpha=r$.

Proof. It suffices to establish that $L_{r} / L_{r-1}=\mathrm{V}\left\{M_{\alpha} \mid \# \alpha=r\right\}$. But if $\alpha \neq \beta$ are admissible $r$-triples, then $K_{\alpha} \cap K_{\beta} \subset L_{r-1}$. The result now follows from the observation that $K_{\alpha} /\left(K_{\alpha} \cap L_{r-1}\right)=M_{\alpha}$.

3. Application to $S O(4)$.

3.1 THEOREM. The space $X=S O(4)$ has $2^{86} \cdot 3^{16}$ homotopy classes of H-structures.

In the course of the proof it will be convenient to refer to the following table of values of $\left[\Sigma^{k} P^{2} ; S^{3}\right]$.

TABLE 3.2

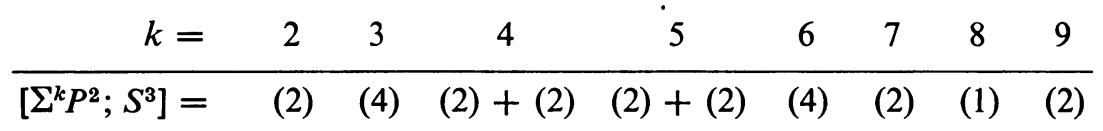


The symbol $(m)$ denotes the abelian group of integers modulo $m$, and + denotes direct sums. The entries are readily deduced from the Appendix to Chapter 12 of Hilton's book [4].

Proof of 3.1. We have $X_{1}=P^{3}=S O(3), X_{2}=S^{3}$. Note that since $X \wedge X$ is simply-connected, $\left[X \wedge X ; S^{3}\right]$ is isomorphic to $\left[X \wedge X ; X_{1}\right]$ under the 2fold covering projection $S^{3} \rightarrow P^{3}$. Let

$$
\begin{aligned}
a & =\#\left[X_{1} \wedge X_{2} \wedge X_{1} \wedge X_{2} ; S^{3}\right]=\#\left[S^{6} \wedge P^{3} \wedge P^{3} ; S^{3}\right], \\
b_{1} & =\#\left[X_{1} \wedge X_{1} \wedge X_{2} ; S^{3}\right]=\#\left[S^{3} \wedge P^{3} \wedge P^{3} ; S^{3}\right], \\
b_{2} & =\#\left[X_{1} \wedge X_{2} \wedge X_{2} ; S^{3}\right]=\#\left[S^{6} \wedge P^{3} ; S^{3}\right] ; \\
c_{1} & =\#\left[X_{1} \wedge X_{1} ; S^{3}\right], \\
c_{2} & =\#\left[X_{1} \wedge X_{2} ; S^{3}\right]=\#\left[S^{3} \wedge P^{3} ; S^{3}\right] \text { and } \\
c_{3} & =\#\left[X_{2} \wedge X_{2} ; S^{3}\right]=\#\left[S^{6} ; S^{3}\right] ;
\end{aligned}
$$

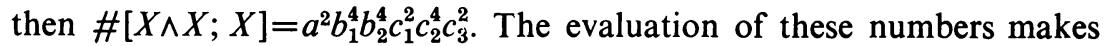
repeated use of Naylor's theorem that $S^{3} \wedge P^{3}=\left(S^{3} \wedge P^{2}\right) \vee S^{6}$ [6]. In addition, we will use Table 3.2 several times in each calculation.

The value of $a$.

Thus

$$
S^{6} \wedge P^{3} \wedge P^{3}=\left(\left(S^{3} \wedge P^{2}\right) \vee S^{6}\right) \wedge\left(\left(S^{3} \wedge P^{2}\right) \vee S^{6}\right) .
$$

$$
a=\#\left[S^{6} \wedge P^{2} \wedge P^{2} ; S^{3}\right] \cdot\left(\#\left[S^{9} \wedge P^{2} ; S^{3}\right]\right)^{2} \cdot \#\left[S^{12} ; S^{3}\right] .
$$

The cofibration sequence of spaces

$$
S^{1} \underset{f}{\longrightarrow} S^{1} \longrightarrow P^{2} \longrightarrow S^{2} \underset{f}{\longrightarrow} S^{2}
$$

in which $f$ ambiguously denotes maps of degree 2 , induces the cofibration sequence

$$
\Sigma^{7} P^{2} \underset{f}{\longrightarrow} \Sigma^{7} P^{2} \longrightarrow S^{6} \wedge P^{2} \wedge P^{2} \longrightarrow \Sigma^{8} P^{2} \underset{f}{\longrightarrow} \Sigma^{8} P^{2}
$$

and hence the exact sequence of groups

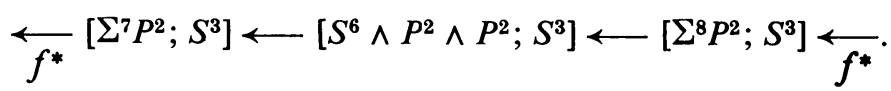

The two outer groups are repetitions and have been omitted from the line above. Since $\left[\Sigma^{7} P^{2} ; S^{3}\right]=Z_{2}$ and $\left[\Sigma^{8} P^{2} ; S^{3}\right]=0$, both homomorphisms $f^{*}$ are trivial, and \#[S $\left[S^{6} \wedge P^{2} \wedge P^{2} ; S^{3}\right]=2$.

On the other hand, \#[ $\left[\Sigma^{9} P^{2} ; S^{3}\right]=2$ and $\#\left[S^{12} ; S^{3}\right]=4$. Thus $a=2^{5}$.

The value of $b_{1}$.

$$
\begin{aligned}
S^{3} \wedge P^{3} \wedge P^{3} & =\left(\left(S^{3} \wedge P^{2}\right) \wedge S^{6}\right) \wedge P^{3}=\left(P^{2} \wedge S^{3} \wedge P^{3}\right) \vee S^{6} \wedge P^{3} \\
& =\left(S^{3} \wedge P^{2} \wedge P^{2}\right) \vee\left(\Sigma^{6} P^{2}\right) \vee\left(\Sigma^{6} P^{2}\right) \vee S^{9}
\end{aligned}
$$


Thus $b_{1}=\#\left[S^{3} \wedge P^{2} \wedge P^{2} ; S^{3}\right] \cdot\left(\#\left[\Sigma^{6} P^{2} ; S^{3}\right]\right)^{2} \cdot \#\left[S^{9} ; S^{3}\right]$. As before, we have an exact sequence

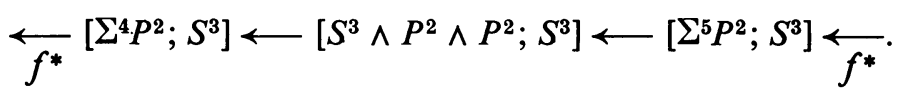

But $\left[\Sigma^{4} P^{2} ; S^{3}\right] \approx\left[\Sigma^{5} P^{2} ; S^{3}\right] \approx Z_{2}+Z_{2}$, and $f^{*}$ is trivial on each of these. This shows \#[ $\left.S^{3} \wedge P^{2} \wedge P^{2} ; S^{3}\right]=2^{4}$.

On the other hand \#[ $\left[\Sigma^{6} P^{2} ; S^{3}\right]=2^{2}$ and $\# \pi_{9}\left(S^{3}\right)=3$, whence $b_{1}=2^{8} \cdot 3$. The values of $b_{2}, c_{1}, c_{2}$ and $c_{3}$.

$$
\begin{aligned}
& b_{2}=\#\left[S^{6} \wedge P^{3} ; S^{3}\right]=\#\left[S^{6} \wedge P^{2} ; S^{3}\right] \cdot \#\left[S^{9} ; S^{3}\right]=2^{2} \cdot 3 ; \\
& c_{1}=\#\left[P^{3} \wedge P^{3} ; S^{3}\right]=2^{8} \cdot 3 \quad[4] ; \\
& c_{2}=\#\left[S^{3} \wedge P^{3} ; S^{3}\right]=\#\left[S^{3} \wedge P^{2} ; S^{3}\right] \#\left[S^{6} ; S^{3}\right]=4 \cdot 12=2^{4} \cdot 3 \\
& c_{3}=\# \pi_{6}\left(S^{3}\right)=2^{2} \cdot 3 .
\end{aligned}
$$

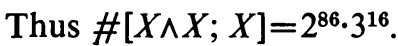

A similar calculation would show that $S^{3} \times S^{3}$ has $2^{20 .} 3^{16}$ homotopy classes of $H$-structures. This result was obtained earlier by Norman [7] using Loibel's Theorem [5].

\section{BIBLIOGRAPHY}

1. A. H. Copeland, Jr., Binary operations on sets of mapping classes, Michigan Math. J. 6 (1959), 7-23. MR 21 \#2232.

2. B. Eckmann and P. J. Hilton, A natural transformation in homotopy theory, Math. Z. 82 (1963), 115-124. MR 27 \#4230.

3. I. M. James, On H-spaces and their homotopy groups, Quart. J. Math. Oxford Ser. (2) 11 (1960), 161-179. MR 24 \#A2966.

4. P. J. Hilton, Homotopy theory and duality, Gordon and Breach, New York, 1965. MR 33 \#6624.

5. G. F. Loibel, Multiplications on products of spheres, An. Acad. Brasil. Ci. 31 (1959), 161-162. MR 21 \#5962.

6. C. M. Naylor, Multiplications on SO(3), Michigan Math. J. 13 (1966), 27-31. MR 32 \#8342.

7. C. W. Norman, Homotopy loops, Topology 2 (1963), 23-43. MR 26 \#4356.

8. R. C. O'Neill, Retracts and retractile subcomplexes, Topology 5 (1966), 191-201. MR 33 \#4931.

Department of Mathematics, University of New Hampshire, Durham, New HAMPSHIRE 03824 